\title{
Evaporation dynamics of a sessile droplet of binary mixture laden with nanoparticles
}

Pallavi Katre

Department of Chemical Engineering, Indian Institute of Technology Hyderabad, Sangareddy 502 285, Telangana, India

Saravanan Balusamy, Sayak Banerjee, and Lakshmana Chandrala

Department of Mechanical and Aerospace Engineering,

Indian Institute of Technology Hyderabad, Sangareddy 502 285, Telangana, India

Kirti Chandra Sahu*

Department of Chemical Engineering, Indian Institute of Technology Hyderabad, Sangareddy 502 285, Telangana, India

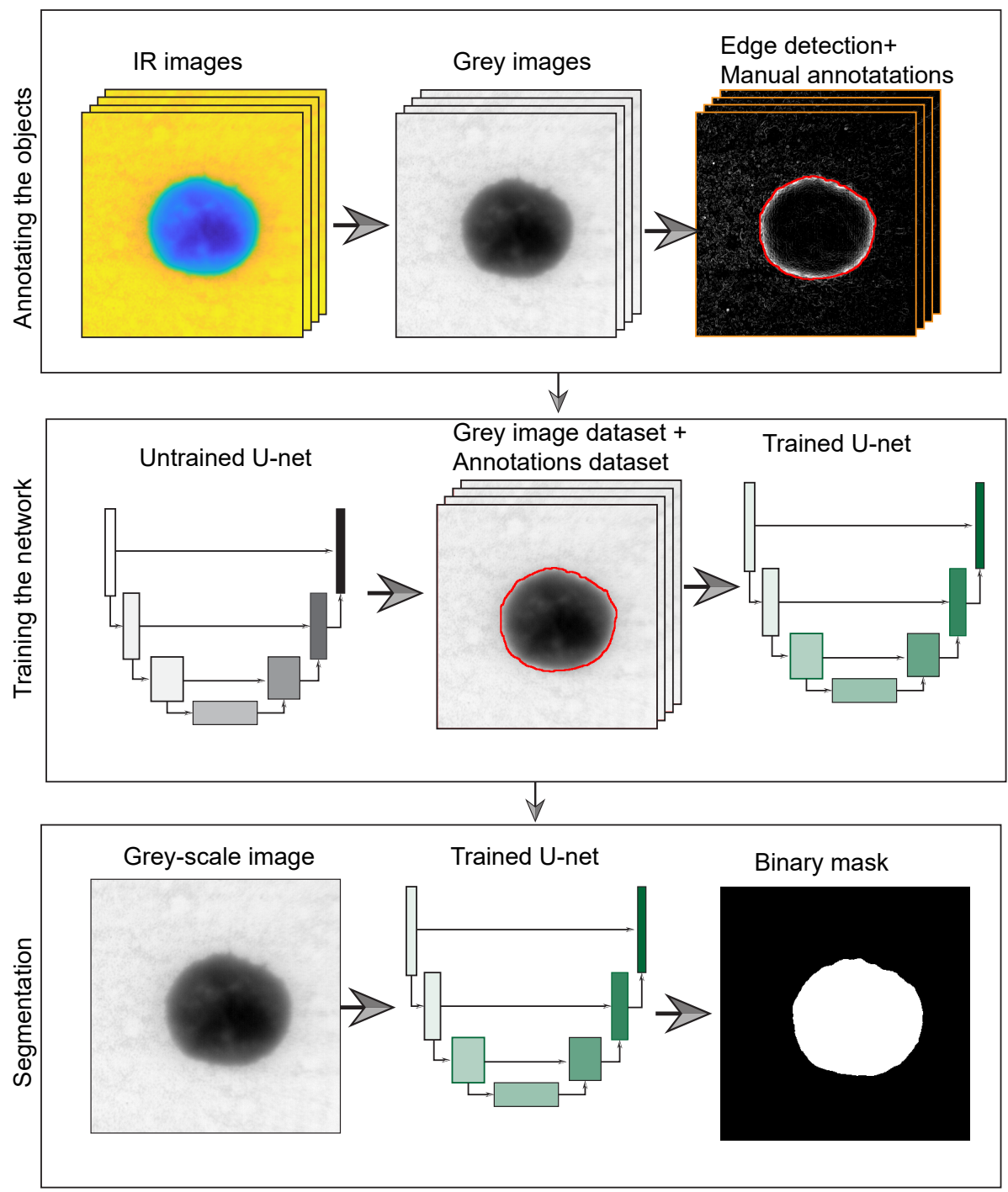

Figure S1: Workflow for machine learning based image segmentation. The flow chart describes the steps involved in segmenting the droplet images such as image annotation, network training, and segmentation.

\footnotetext{
*ksahu@che.iith.ac.in
} 


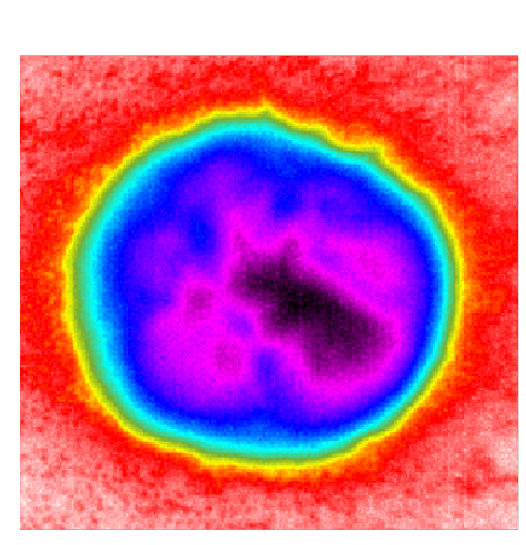

(a) Raw image

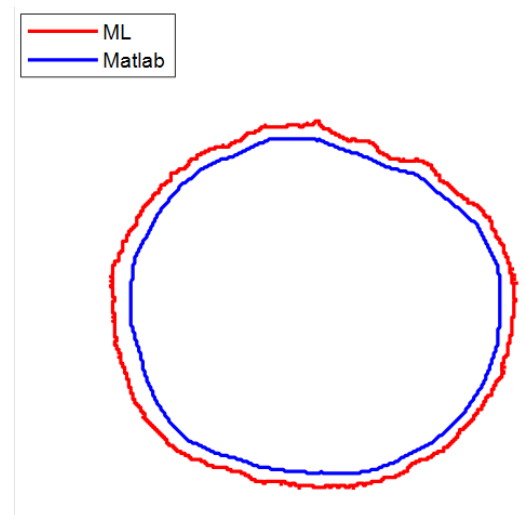

(b) Contour profile

Figure S2: A comparison of post-processed images using the conventional MATLAB ${ }^{\circledR}$ program and machine learning (ML) code based on U-Net architecture.

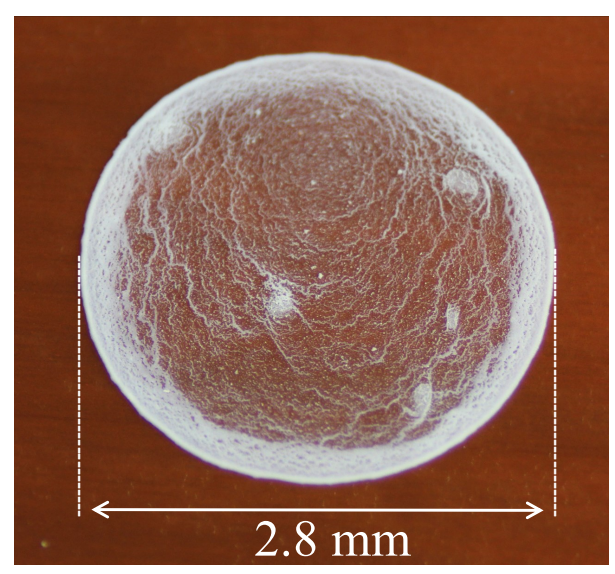

Figure S3: Deposition pattern observed in the case of a (E 50\% + W 50\%) droplet with 0.6 wt.\% nanoparticle loading at $T_{s}=60^{\circ} \mathrm{C}$.

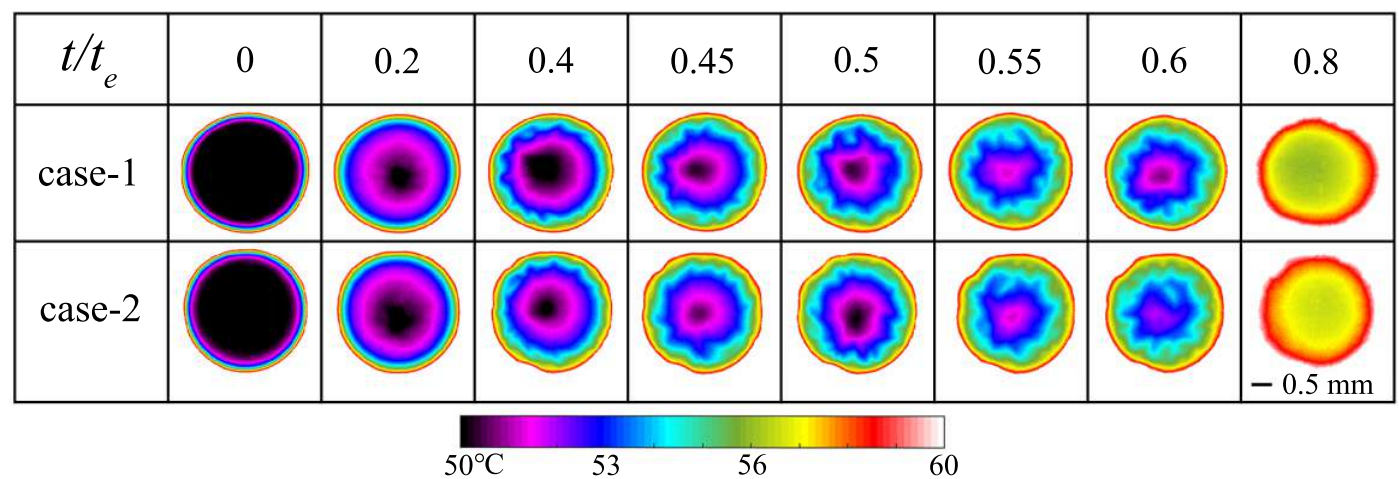

Figure S4: Universal behavior of (E $80 \%+\mathrm{W} 20 \%$ ) droplet with nanoparticle loading of 0.6 wt. $\%$ at $T_{s}=60^{\circ} \mathrm{C}$. 
Table S1: The properties of the materials used.

\begin{tabular}{|c|c|c|c|c|c|}
\hline \multirow{2}{*}{ Properties } & \multicolumn{2}{|c|}{ Water } & \multicolumn{2}{c|}{ Ethanol } & $\mathrm{Al}_{2} \mathrm{O}_{3}$ \\
\cline { 2 - 6 } & $50^{\circ} \mathrm{C}$ & $60^{\circ} \mathrm{C}$ & $50^{\circ} \mathrm{C}$ & $60^{\circ} \mathrm{C}$ & - \\
\hline Density $\left(\mathrm{Kg} / \mathrm{m}^{3}\right)$ & 988 & 983 & 758 & 747 & 3950 \\
\hline Diffusivity $\left(\mathrm{m}^{2} / \mathrm{s}\right)$ & $2.71 \times 10^{-5}$ & $2.84 \times 10^{-5}$ & $1.24 \times 10^{-5}$ & $1.304 \times 10^{-5}$ & - \\
\hline Kinematic viscosity $\left(\mathrm{m}^{2} / \mathrm{s}\right)$ & $5.54 \times 10^{-7}$ & $4.07 \times 10^{-7}$ & $9.0 \times 10^{-7}$ & $8.0 \times 10^{-7}$ & - \\
\hline Thermal conductivity $(\mathrm{W} / \mathrm{mK})$ & 0.64 & 0.65 & 0.162 & 0.159 & - \\
\hline Molecular weight $(\mathrm{g} / \mathrm{mol})$ & \multicolumn{2}{|c|}{18.02} & \multicolumn{2}{|c|}{46.07} & 101.96 \\
\hline
\end{tabular}

